CASE REPORT

\author{
T.Y. Jeon \\ H.-J. Kim \\ S.T. Kim \\ T.-Y. Chung \\ C. Kee
}

\section{MR Imaging Features of Giant Reservoir Formation in the Orbit: An Unusual Complication of Ahmed Glaucoma Valve Implantation}

\begin{abstract}
SUMmARY: We report the MR imaging findings of an unusually large fluid collection, so-called giant reservoir, around an Ahmed glaucoma valve implant that caused progressive proptosis in a patient with posttraumatic glaucoma. Although other cystic lesions of the juxtalacrimal area of the orbit, such as dermoid cyst, can present similar imaging findings, the characteristic morphology and location should lead the radiologist to the correct nature of this cystic lesion.
\end{abstract}

G aucoma drainage devices provide an alternative treatment in complicated and refractory glaucoma cases. ${ }^{1,2}$ The Ahmed glaucoma valve (AGV) implant (New World Medical, Rancho Cucamonga, Calif) is 1 of the common ocular tube devices currently used for this surgical technique. To prevent ocular hypotony, it has a unidirectional valve designed to offer a set resistance to the aqueous outflow from the anterior chamber with an intraocular pressure of $8 \mathrm{~mm} \mathrm{Hg}$ or less. ${ }^{3,4}$ The aqueous humor forms a fibrous encapsulated bleb on the endplate of the implant, which is resorbed through intercellular spaces and the lymphatic system of the adjacent periocular tissues. Sometimes this bleb becomes unusually large, and a so-called giant reservoir is formed, which can simulate a cystic lesion in the orbit if the ophthalmologic history is not known. We present the MR imaging features of the giant reservoir formed after AGV implantation that caused progressive proptosis in a patient with posttraumatic glaucoma.

\section{Case Report}

A 22-year-old man presented with a progressive proptosis and pain of the right eye noticed for 1 week. At 14 years of age, he suffered a blunt injury in the right eye necessitating penetrating keratoplasty. Six years later, secondary glaucoma developed, which was refractory to a 3-month trial of meticulous medical treatment, and AGV implantation was performed as described by Coleman et al. ${ }^{5}$ During the $30-$ month follow-up, until recently, the intraocular pressure had been well maintained below $21 \mathrm{~mm} \mathrm{Hg}$ by antiglaucoma and antifibrotic medications coupled with digital massage.

An ophthalmologic examination revealed the hyperemic conjunctiva and the peripherally shallow anterior chamber in the right eye. The patient could only count fingers from a distance of $2 \mathrm{ft}$, when using only the right eye. Left eye evaluation using the standard $\mathrm{E}$ chart showed a normal visual acuity of 20/20. The right and left intraocular pressures measured by the Goldmann applanation tonometry were 28-30 $\mathrm{mm} \mathrm{Hg}$ and $14 \mathrm{~mm} \mathrm{Hg}$, respectively. Axial biometry readings were 17.5-mm OD and 15-mm OS.

For identifying the cause of progressive proptosis, MR examination of the orbit was performed using a 3T scanner (Achieva; Philips Medical

Received January 18, 2007; accepted after revision February 1.

From the Departments of Radiology (T.Y.J., H.-J.K., S.T.K.) and Ophthalmology (T.-Y.C., C.K.), Samsung Medical Center, Sungkyunkwan University School of Medicine, Seoul, Korea.

Please address correspondence to Hyung-Jin Kim, Department of Radiology, Samsung Medical Center, Sungkyunkwan University School of Medicine, 50 Ilwon-Dong, KangnamKu, Seoul 135-710, Korea; e-mail: hyungkim@smc.samsung.co.kr

DOI 10.3174/ajnr.A0610
Systems, Best, the Netherlands), which revealed a $1.7-\mathrm{cm}$ ovoid, thinwalled, cystic lesion in the superotemporal aspect of the right orbit between the lacrimal gland and the globe (Fig 1). The lesion was apparently partitioned by the centrally located curvilinear band showing very dark signal intensity on both T1- and T2-weighted images (Fig $1 A,-B$ ). The lesion caused mild indentation of the adjacent superotemporal wall of the globe, which was mildly displaced anteroinferiorly. These MR imaging appearances were thought to correspond with a large fluid collection, so-called giant reservoir, surrounding the endplate of the AGV implant.

\section{Discussion}

Glaucoma drainage implants are being increasingly used in recent years and have been used successfully for the treatment of complicated glaucomas, including neovascular glaucoma, aphakic and pseudoaphakic glaucoma, postpenetrating keratoplasty glaucoma, pediatric glaucoma, and uveitic glaucoma. ${ }^{6,7}$ The implants currently in common use include the AGV implant, the Baerveldt glaucoma implant (Advanced Medical Optics, Santa Ana, Calif), the Krupin slit valve (Hood Laboratories, Pembroke, Mass), and the Molteno implant (Molteno Ophthalmic, Dunedin, New Zealand).

The AGV implant, first introduced in 1993, consists of a drainage tube made of silicone and a scarab-shaped endplate made of either polypropylene or silicone (Fig 2). Fenestrations have been added to the plate of the silicone models. Different sizes $\left(96,184\right.$, and $\left.364 \mathrm{~mm}^{2}\right)$ according to the surface area of the endplate are commercially available. ${ }^{2}$ Implants with a larger surface area and a thin capsule at the endplate will have a greater effect in lowering the patient's intraocular pressure. The endplate rests on the scleral surface after an incision of the conjunctiva and Tenon capsule, usually at the superior equator of the globe between the superior and lateral rectus muscles. The AGV is designed to minimize postoperative hypotony by adding a valve mechanism to an aqueous shunt surgery device to maintain a more predictable intraocular pressure. Moreover, the tube dose not require occlusion with a ligature or obturator, thus simplifying the initial surgery and eliminating the need for an additional procedure to remove the obturator. ${ }^{8}$

AGV implantation carries a diverse range of postoperative complications. ${ }^{9}$ The early complications include transient hypotony, shallow anterior chamber, tube blockage, and hemorrhage. The late complications are encapsulated bleb formation, exposure and malposition of tube, corneal decompression, extrusion of implant, and fibrotic reaction around the valve.

An encapsulated bleb (also called a Tenon cyst) is a common postoperative complication after AGV implantation, which de- 

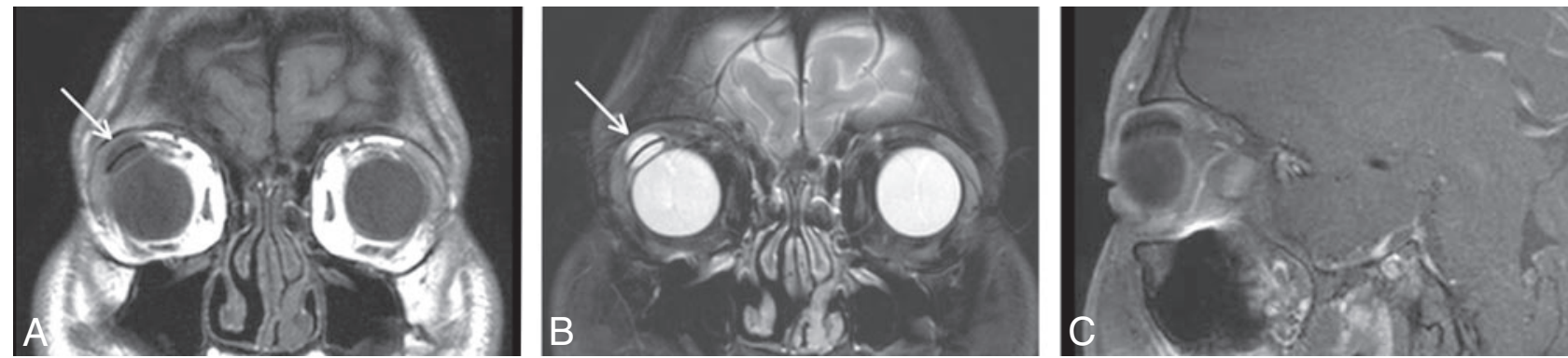

Fig 1. A 22-year-old man with giant reservoir associated with Ahmed glaucoma valve implantation. $A$ and $B$, Coronal T1-weighted $(A)$ and fat-suppressed T2-weighted ( $B$ ) MR images of the orbit show a 1.7-cm ovoid, thin-walled, purely cystic lesion in the superotemporal aspect of the right orbit between the lacrimal gland and the globe (arrows). A dark curvilinear band representing the silicone-made endplate of the implant is seen at the center of the cyst on both T1-and T2-weighted images. Also noted is mild indentation of the adjacent superotemporal wall of the right globe. $C$, Axial contrast-enhanced fat-suppressed T1-weighted MR image demonstrates no significant enhancement within the lesion.
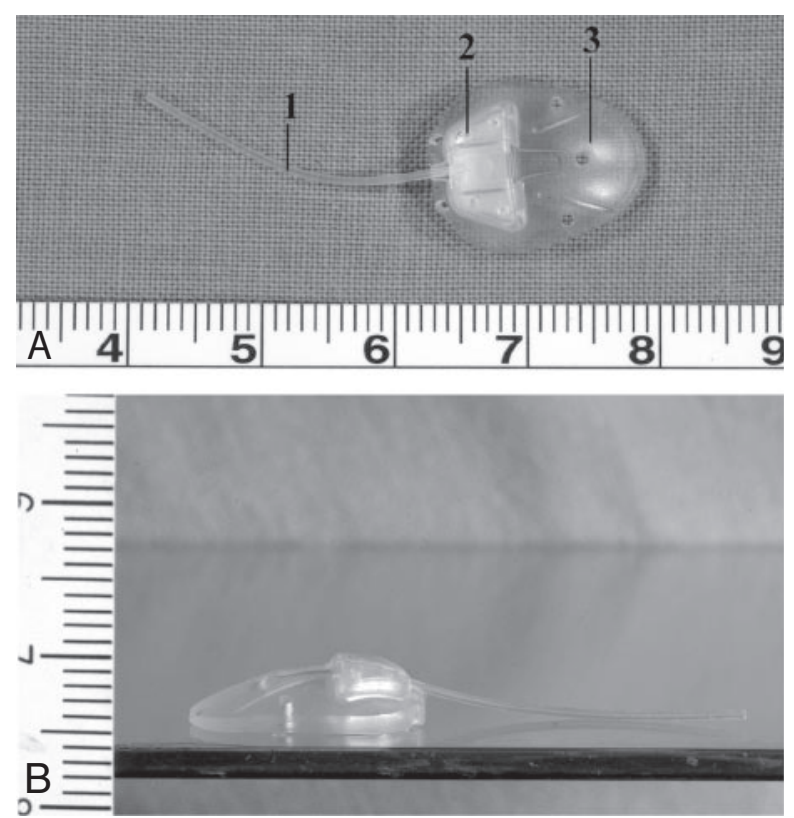

Fig 2. Ahmed glaucoma valve implant with a surface area of $184 \mathrm{~mm}^{2}$. A, Photograph shows the implant composed of 3 parts: 1) drainage tube, 2) valve, and 3) endplate. $B$, Photograph of the side of the implant shows the crescentic shape of the endplate with a thickness of $0.9 \mathrm{~mm}$.

velops when Tenon's capsule adheres to the episcleral space forming a high, domed, smooth, 2-layered bleb. ${ }^{4}$ The encapsulated bleb is impervious to the aqueous humor, which results in intraocular pressure elevation. ${ }^{10,11}$ The incidence of bleb encapsulation is much higher with the AGV than the other glaucoma drainage implants and has been estimated to be between $40 \%$ and $80 \%,{ }^{12}$ which might be attributed to certain factors of the aqueous humor that stimulate a fibrotic response. Tsai et $\mathrm{al}^{13}$ have suggested that immediate aqueous filtration with inflammatory factors may stimulate the fibrotic response in the subconjunctival space when the Ahmed implant is used, and delayed flow with a ligated, nonvalved implant may elicit a less fibrous reaction. The rate of bleb encapsulation has also been reported to be related to differences in the biomaterial, shape, and consistency of the endplate. $^{14}$

Sometimes as seen in this case, an unusually large encapsulated bleb, so-called giant reservoir, is formed around the endplate of the AGV implant and can clinically produce proptosis and radiologically mimic other cystic lesions of the orbit, such as a dermoid cyst, which is particularly common around the lacri- mal gland fossa. The diagnosis can be even more difficult if the ophthalmologic history is not known. On MR imaging, the giant reservoir associated with AGV implantation is seen as a thinwalled ovoid cyst located at the superotemporal aspect of the orbit near the lacrimal gland fossa. The wall of the cyst is probably formed by the fibrous pseudocapsule. The silicone-made endplate of the implant is seen as a very dark band located at the center of the cyst on both T1- and T2-weighted MR images. Although digital massage and antifibrotic medication can effectively reduce the size of the reservoir in most cases, surgical removal of scar tissues should be considered in resistant cases. ${ }^{2}$

In conclusion, we report the MR imaging findings of the giant reservoir formed after AGV implantation in a patient with posttraumatic glaucoma. Although other cystic lesions, such as dermoid cyst, can present similar imaging findings, the characteristic morphology and location should lead the radiologist to the correct nature of this cystic lesion.

\section{References}

1. Englert JA, Freedman SF, Cox TA. The Ahmed valve in refractory pediatric glaucoma. Am J Ophthalmol 1999;127:34-42

2. Schwartz KS, Lee RK, Gedde SJ. Glaucoma drainage implants: a critical comparison of types. Curr Opin Ophthalmol 2006;17:181-89

3. Djodeyre MR, Peralta-Calvo J, Abelairas-Gomez J. Clinical evaluation and risk factors of time to failure of Ahmed glaucoma valve implant in pediatric patients. Ophthalmology 2001;108:614-20

4. Lai JS, Poon AS, Chua JK, et al. Efficacy and safety of the Ahmed glaucoma valve implant in Chinese eyes with complicated glaucoma. $\mathrm{Br}$ J Ophthalmol 2000;84:718-21

5. Coleman AL, Hill R, Wilson MR, et al. Initial clinical experience with the Ahmed glaucoma valve implant. Am J Ophthalmol 1995;120:23-31

6. De Leon-Ortega JE, Girkin CA. Ocular trauma-related glaucoma. Ophthalmol Clin North Am 2002;15:215-23

7. Hitchings R. Traumatic glaucoma. J Glaucoma 2001;10:436-39

8. Setabutr P, Bell NP, Feldman RM. Intraoperative management of nonfunctioning Ahmed glaucoma valve implant. Ophthalmic Surg Lasers Imaging 2006;37:62-64

9. Chen $\mathrm{H}$, Zhang SX, Liu L, et al. Intermediate-term and long-term clinical evaluation of the Ahmed glaucoma valve implantation. Zhonghua Yan $\mathrm{Ke} \mathrm{Za}$ Zhi 2005;41:796-802

10. Ayyala RS, Layden WE, Slonim CB, et al. Anatomic and histopathologic findings following a failed Ahmed glaucoma valve device. Ophthalmic Surg Lasers 2001;32:248-49

11. Eibschitz-Tsimhoni M, Schertzer RM, Musch DC, et al. Incidence and management of encapsulated cysts following Ahmed glaucoma valve insertion. $J$ Glaucoma 2005; 14:276-79

12. Hong $\mathrm{CH}$, Arosemena A, Zurakowski D, et al. Glaucoma drainage devices: a systematic literature review and current controversies. Surv Ophthalmol 2005;50:48-60

13. Tsai JC, Johnson CC, Dietrich MS. The Ahmed shunt versus the Baerveldt shunt for refractory glaucoma: a single-surgeon comparison of outcome. Ophthalmology 2003;110:1814-21

14. Ayyala RS, Michelini-Norris B, Flores A, et al. Comparison of different biomaterials for glaucoma drainage devices: part 2. Arch Ophthalmol 2000;118:1081-84 